\title{
The Impact of Tryptophan Depletion and 5-HTTLPR Genotype on Passive Avoidance and Response Reversal Instrumental Learning Tasks
}

\author{
Elizabeth C Finger*,1,2, Abigail A Marsh',2, Beata Buzas ${ }^{3}$, Niveen Kamel ${ }^{1,2}$, Rebecca Rhodes ${ }^{4}$, \\ Meena Vythilingham ${ }^{1,2}$, Daniel S Pine ${ }^{1,2}$, David Goldman ${ }^{3}$ and James R Blair ${ }^{1,2}$ \\ 'Mood and Anxiety Disorders Program, Unit on Affective Cognitive Neuroscience, National Institute of Mental Health, Bethesda, MD, USA; \\ ${ }^{2}$ Department of Health and Human Services, National Institutes of Health, Bethesda, MD, USA; ${ }^{3}$ Laboratory of Neurogenetics, National Institute \\ on Alcohol Abuse and Alcoholism, National Institutes of Health, Bethesda, MD, USA; ${ }^{4}$ Psychiatry Group, MRC Clinical Sciences Centre, Imperial \\ College, Hammersmith Hospital, London, UK
}

\begin{abstract}
Transient reductions in serotonin levels during tryptophan depletion (TD) are thought to impair reward processing in healthy volunteers, while another facet of the serotonergic system, the serotonin transporter (5-HTTLPR) short allele polymorphism, is implicated in augmented processing of aversive stimuli. We examined the impact and interactions of TD and the serotonin promoter polymorphism genotype on reward and punishment via two forms of instrumental learning: passive avoidance and response reversal. In this study, healthy volunteers $(n=35)$ underwent rapid TD or control procedures and genotyping $(n=26)$ of the 5 -HTTLPR for long and short allele variants. In the passive avoidance task, tryptophan-depleted volunteers failed to respond sufficiently to rewarded stimuli compared to the control group. Additionally, long allele homozygous individuals $(n=1 \mathrm{I})$ were slower to learn to avoid punished stimuli compared to short allele carriers $(n=15)$. TD alone did not produce measurable deficits in probabilistic response reversal errors. However, a significant drug group by genotype interaction was found indicating that in comparison to short allele carriers, tryptophan-depleted individuals homozygous for the long allele failed to appropriately use punishment information to guide responding. These findings extend prior reports of impaired reward processing in TD to include instrumental learning. Furthermore, they demonstrate behavioral differences in responses to punishing stimuli between long allele homozygotes and short allele carriers when serotonin levels are acutely reduced.

Neuropsychopharmacology (2007) 32, 206-2 15. doi:I0.1 038/sj.npp. I 30 I I82; published online 9 August 2006
\end{abstract}

Keywords: tryptophan depletion; 5-HTTLPR; reward; response reversal

\section{INTRODUCTION}

Abnormalities of serotonergic systems are implicated in several psychiatric disorders with diverse psychopathology (Rogers et al, 1999; Stockmeier, 1997; Zohar et al, 2004). While serotonergic activity is thought to be functionally reduced in many of these disorders, the specific contributions serotonin makes to cognition and affect remain unclear. Tryptophan depletion (TD), a procedure which transiently lowers central nervous system serotonin levels by reducing serum and central nervous system levels of its

*Correspondence: Dr EC Finger, Unit on Affective Cognitive Neuroscience, Mood and Anxiety Disorders Program, National Institute of Mental Health, I5K, North Drive, MSC 2670, Bethesda, MD 20892, USA, Tel: + I 30I 402 6856, Fax: + I 30I 4026100 ,

E-mail: fingere@mail.nih.gov

Received 27 March 2006; revised 16 June 2006; accepted 6 July 2006 Online publication: 12 July 2006 at http://www.acnp.org/citations/ Npp07|206060199/default.pdf precursor tryptophan (Carpenter et al, 1998; Gessa et al, 1975; Perez-Cruet et al, 1974; Williams et al, 1999), offers a means to elucidate the roles of this neurotransmitter (Park et al, 1994). Characterization of associations between alleles of the serotonin transporter, in particular two functional polymorphisms found within the serotonin transporter promoter region (5-HTTLPR), and task performance (Clark et al, 2005) or neural activity (Hariri et al, 2005; Pezawas et al, 2005), offers another means to assess the role of this neurotransmitter. In this study, we used TD and genetic assay of the long (L) and short (S) alleles of the 5-HTTLPR. Our aim was to determine the impact of a hyposerotonergic state and a LL-homozygous relative to S-allele carrier state, as well as interactions of these variables, on instrumental learning as indexed by passive avoidance learning, and response reversal performance.

To date, investigations of the effects of TD on processing of reinforcement information have identified deficits in processing of reward information, while studies of the 
effects of TD on emotional stimuli have focused on deficits in processing of negative facial expressions. TD has been reported to lead to alter reward processing in decisionmaking tasks. Thus, Rogers et al (1999) found reduced discrimination between magnitudes of expected gains in a gambling paradigm while Cools et al (2005b) found reduced reward related speeding of response times in a cuedreinforcement reaction time task (Cools et al, 2005b; Rogers et al, 1999). In contrast, several studies have found no effect on the processing of punishment information in gambling paradigms (Anderson et al, 2003; Rogers et al, 2003; Talbot et $a l, 2005)$. A role for serotonin in learning is further supported by a recent report of impaired probabilistic learning following acute administration of the SSRI citalopram to healthy volunteers (Chamberlain et al, 2006). Studies investigating the processing of emotional stimuli in healthy volunteers have found TD-induced impairments in recognition of fearful facial expressions (Harmer et al, 2003). These findings are also are supported by SSRI challenge studies which have found reduced recognition of negative emotional expressions (Harmer et al, 2004) and decreased bold responses in the amygdala and medial PFC during viewing of fearful faces (Harmer et al, 2006) following citalopram administration.

Few studies have investigated the potential impact of the serotonin transporter polymorphism on processing of reinforcement information. Neuroimaging studies indicate that 5-HTTLPR status impacts response to social reinforcers. LL-homozygous individuals demonstrate reduced neural responses to fearful or angry faces compared to S-carriers (Canli et al, 2005; Hariri et al, 2005; Hariri et al, 2002; Heinz et al, 2005). These studies have focused on the processing of negative affective stimuli, although of note, of the two of the studies also used positive affective stimuli, one identified differences in the processing of positive facial expressions in S-carriers (Canli et al, 2005).

The base for the differential effects of acute TD and serotonin transporter polymorphisms has yet to be fully elucidated. Acute TD results in an acute reduction in CSF levels of serotonin metabolites (Carpenter et al, 1998; Williams et al, 1999), suggesting decreases in extracellular serotonin availability due to reduction of precursor. In contrast, behavioral or neural response differences due to serotonin transporter genotype likely reflect not only current differences in transporter levels, but also neurodevelopmental and downstream effects of chronic differences in levels of transporter expression (cf. Hariri and Holmes, 2006).

In an attempt to further define and clarify the effects of TD and 5-HTTLPR status on reward and punishment, and in particular, stimulus-reinforcement learning, we employed an instrumental learning task, the passive avoidance task (Newman and Kosson, 1986). This task was originally modeled after go-no-go instrumental learning paradigms in rodents, and has the advantages of a well-characterized neural circuitry. Electrophysiologic and lesion studies in animals have demonstrated the involvement of orbitofrontal cortex, insula, striatum, hippocampus, and amygdala in passive avoidance learning (Ambrogi Lorenzini et al, 1997, 1999; Bermudez-Rattoni and McGaugh, 1991; Gallagher et al, 1999; McGaugh, 2002; Sandberg et al, 1984; Schoenbaum et al, 1998; Treit and Menard, 1997; Tremblay and Schultz, 2000). Recruitment of analogous regions in humans during passive avoidance learning has recently been confirmed by an fMRI investigation, with correct responses recruiting rostral anterior cingulate, insula, caudate, hippocampal regions, and the amygdala (Kosson et al, 2006). To date, no studies have assessed the effect of TD or the association of serotonergic genotypes on passive avoidance learning performance in humans. Based on the prior suggestions of diminished reward sensitivity, we predicted that tryptophan-depleted participants would make more omission errors (failures to respond to stimuli associated with reward). Prior studies have reported that $\mathrm{S}$-carriers are hypersensitive to threat as they more rapidly develop conditioned fear responses (Garpenstrand et al, 2001) and demonstrate heightened amygdala BOLD signal in response to social threat stimuli (Hariri et al, 2005; Heinz et al, 2005). Based on these findings, we predicted that the S-carriers would be more sensitive to punishment information and more avoidant of stimuli associated with punishment than the LL-homozygotes during passive avoidance learning.

The role of serotonin in response reversal has been inconclusive to date, with reports of both normal performance and deficits in response reversal in the setting of TD. In favor of serotonergic mediation of response reversal, two studies have reported increased errors in reversal and dimensional shift phases of an ID/ED task in the lowtryptophan group (Park et al, 1994; Rogers et al, 1999). This finding is supported by animal studies of response reversal impairments after selective ablation of prefrontal serotonergic neurons (Clarke et al, 2004, 2005). The role for serotonin in response reversal is not clear, however, as a recent study using the same ID/ED paradigm in tryptophan-depleted volunteers found no impairment in reversal learning or set shifting (Talbot et al, 2005). Additionally, in probabilistic response reversal tasks two other studies have found no significant behavioral effects of TD on reversal errors, although both studies did describe global increases in reaction times in the tryptophan-depleted groups (Evers et al, 2005; Murphy et al, 2003). It is important to note that the role of 5-HTTLPR alleles on response reversal learning has not been assessed. It is possible that the discrepant results described above may reflect different representations of LL- or S-allele carriers in the participant groups across studies.

To test the hypothesis that response reversal deficits may result from a hyposerotonergic state and interactions with 5-HTTLPR alleles, we administered the revised probabilistic response reversal task (Budhani and Blair, 2005). Unlike the older probabilistic response reversal paradigm where the same pair serially reverses throughout the task (Cools et al, 2002; Evers et al, 2005; Kringelbach and Rolls, 2003; O'Doherty et al, 2003; Remijnse et al, 2005), in the revised PRR paradigm the participant learns about multiple stimulus pairs which only reverse once, and nonreversing control pairs. Serial reversals of a single stimulus pair are problematic as it becomes difficult to disentangle acquisition and reversal trials (acquisition of novel stimuli occurs only once). We predicted that examination of the impact of 5-HTTLPR genotype on response reversal performance and interactions with TD would unmask TD-induced deficits in response reversal performance. 


\section{METHODS}

\section{Participants}

In total 35 healthy volunteers underwent a screening visit at the National Institutes of Mental Health which included a medical history and physical exam performed by a physician, a Structured Clinical Interview for DSM-IV performed by a clinician, and blood and urine screening tests. The matrix reasoning and verbal subtests of the Wechsler Abbreviated Scale of Intelligence were administered to obtain an estimated IQ score. Participants were free of any medical illness, current Axis I disorders, past major affective disorder or psychosis, had no first-degree family members with a known or suspected history of depression, and currently were taking no psychotropic medications. All volunteers gave written consent and were paid for their participation. After randomization, 16 subjects (seven males and nine females) received the tryp-capsules; 19 participants (10 males and nine females) received placebo capsules. No participants reported nausea. Only one subject (who received placebo) reported any subjective mood change (mild anxious feeling) during the study visit. ANOVAs revealed no significant group differences in mean age $(\mathrm{F}(1,34)=0.26, p=0.6), \mathrm{M}[$ tryp- $]=27.25$ years, $\mathrm{SE}$ $1.88 ; \mathrm{M}[$ placebo] $=28.63$ years, SE 1.91 , IQ $(p=0.7)$, $\mathrm{M}[$ tryp -$]=114.8, \mathrm{SE} 2.66, \mathrm{M}[$ placebo $]=113.2, \mathrm{SE} 2.80$, or in VAS mood ratings $(\mathrm{F}(1,31)=2.16, p=0.15)$.

\section{Study Design}

In a double blind, placebo controlled, parallel group design to minimize practice and order effects, participants were instructed to observe a low-tryptophan diet (10-15 g of protein) for $24 \mathrm{~h}$ preceding their study visit and to fast from midnight prior to the study day. On the morning of the study visit participants were randomized to receive either 70 TD capsules (tryp-) containing $4.2 \mathrm{~g} \mathrm{~L}$-isoleucine, $6.6 \mathrm{~g}$ L-leucine, $4.8 \mathrm{~g}$ L-lysine, $1.5 \mathrm{~g}$ L-methionine, $6.6 \mathrm{~g}$ L-phenylalanine, $3.0 \mathrm{~g}$ L-threonine, and $4.8 \mathrm{~g} \mathrm{~L}$-valine, or 70 placebo capsules containing $31.5 \mathrm{gm}$ of lactose (Wolfe et al, 1995). This protocol was selected as it had previously been used in our institute and succeeded in minimizing side effects of nausea while producing reductions in free tryptophan levels comparable to studies using larger doses of amino acids and balanced mixtures (Neumeister et al, 2004). Serum was drawn on admission and $5 \mathrm{~h}$ after ingestion of the last capsule for analysis of free tryptophan and total tryptophan: large neutral amino acids (LNAA) ratios. Serum was also collected on admission for 5-HTTLPR genetic analysis. After capsule ingestion, a low-tryptophan breakfast and lunch (containing a total of $60 \mathrm{mg}$ of tryptophan) were served. Mood changes were monitored by visual analog scales and verbal report. At $5 \mathrm{~h}$ after the last capsule ingestion volunteers completed a battery of neuropsychological tasks within $90 \mathrm{~min}$, which included the passive avoidance and probabilistic response reversal tasks.

\section{Passive Avoidance Learning Task}

The passive avoidance task was a modified version of Newman and Kosson's (1986) task (Blair et al, 2004). Stimuli were 12 white 2-digit numbers presented for
$3000 \mathrm{~ms}$ sequentially on a black background. Six of the stimuli, the S+s, were 'good' stimuli; an approach (bar press) response to these stimuli led to the participant gaining points. Six of the stimuli, the $S-s$, were 'bad' stimuli; the participant learns to avoid these stimuli as an approach (bar press) response to them led to the participant losing points. The point levels included a graded reward/ punishment schedule $( \pm 1,400,800,1200,1600$, or 2000 points) to assess any effects produced by different levels of reward or punishment. Participants learned by trial and error to click on the mouse button to the $S+$ and to refrain from responding to the $\mathrm{S}-$. After each response, participants received feedback on points they had won or lost. If no response was made, a blank screen appeared in place of feedback. Stimuli were presented once per block for 10 blocks per session. Performance was assessed by analysis of omission errors (failure to respond to a rewarded stimulus) and passive avoidance errors (response to a punished stimulus).

\section{Probabilistic Response Reversal Task}

The probabilistic response reversal task administered was based on that previously described by (Budhani and Blair, 2005). Stimuli were assigned into pairs randomly at the beginning of the task, and remained in the same pairs throughout the task. Stimuli comprised 12 line drawings of animals (Snodgrass and Vanderwart, 1980), each shaded in a different color. Stimuli measured $4 \times 4 \mathrm{~cm}$ and were presented on a gray background. On each trial stimuli were presented in pairs on the screen. Stimulus locations were assigned randomly on each trial to one of 16 screen locations. Participants chose one of the stimuli by clicking on it with the mouse, after which they received either positive ('you win 100 points') or negative ('you lose 100 points') feedback on the basis of the reinforcement contingency of that pair. One of the animals in each pair was always more likely than the other to be rewarded rather than punished. Participants began the task with 0 points. A running total of points was presented at the bottom of the screen after each trial. Trials were self-paced.

The reinforcement contingencies were probabilistic such that the 'correct' pair was not always rewarded and the 'incorrect' pair was not always punished. The 'correct' stimulus in a pair with an 80-20 reward-punishment contingency was rewarded on 8 out of every 10 trials and punished on 2 out of every 10 trials. Conversely, the 'incorrect' stimulus was punished on 8 out of every 10 trials and rewarded on 2 out of every 10 trials. The order of probabilistic feedback was randomized within the program.

There were six different pairs of stimuli: two test pairs which changed contingency (reversing pairs) and four 'dummy' pairs which did not (non-reversing pairs). The two reversing pairs had contingencies $100-0$ and $80-20$. The reinforcement contingency of the reversing pairs remained constant for 40 trials (phase 1: acquisition of the discrimination). Upon completing 40 trials the reinforcement contingency the reversing pairs was reversed (phase 2: reversal of the discrimination), for a total of 80 trials per stimulus pair. Thus the previously correct stimulus became the incorrect stimulus and the previously incorrect stimulus now became the correct stimulus. Three of the nonreversing 
dummy pairs had a contingency of 100-0, the fourth nonreversing pair had a contingency of 80-20.

Performance was assessed by errors to criterion (criterion defined as six consecutive correct responses) and total errors in the acquisition and reversal phases. Attainment of criterion in the acquisition phase was assessed to assure proper learning of the stimulus-reinforcement associations so that reversal learning could be assessed.

\section{Biochemical Analysis}

Serum was collected in prechilled EDTA tubes and immediately following collection was centrifuged for $15 \mathrm{~min}$ at 300 r.p.m. and $4^{\circ} \mathrm{C}$. They were subsequently stored at $-70^{\circ} \mathrm{C}$. Plasma TRP concentrations were determined by reverse-phase High Performance Liquid Chromatography (HPLC) in conjunction with fluorescence end-point detection. For total TRP plasma proteins were removed by precipitation with $3 \%$ trichloroacetic acid followed by centrifugation. For the estimation of free TRP, protein bound TRP was separated from free by filtration through $10 \mathrm{~K}$ cutoff microfilters via a centrifugation process. LNAAs were analyzed via gradient HPLC with utilization of precolumn derivatization and fluorescence end-point detection.

\section{Genotype Analysis}

DNA extraction. White blood cells were isolated from $10 \mathrm{ml}$ whole blood using a Ficoll gradient method and stored frozen at $-20^{\circ} \mathrm{C}$. DNA was extracted from the previously frozen white blood cell pellets using the Versagene DNA extraction kit (Gentra) following the manufacturer's protocol.

Allelic discrimination. The S- and L-alleles at the 5HTTLPR were determined using a $5^{\prime}$-exonuclease assay as described (Hu et al, 2006). Briefly, the forward (GCAACCTCCCAGCAACTCCCTGTA) and reverse PCR primers (GAGGTGCAGGGGGATGCTGGAA) amplify a $182 \mathrm{bp}$ for the L-allele and a $138 \mathrm{bp}$ amplicon for the
S-allele. Allelic discrimination (ADP) was performed using a 5 '-VIC labeled probe (VIC-TCCCCCCCTTCACCCCTCG CGGCATCC) complimentary to the sequence within the $43 \mathrm{bp}$ insertion of the L-allele. A labeled internal control probe (ICP) (FAM-TGCAGCCCCCCCAGCATCTCCC) complimentary to a single copy sequence present in both the $\mathrm{L}$ - and $\mathrm{S}$-alleles was included to detect the S-allele. The reaction was carried out in a $25 \mu \mathrm{l}$ volume containing $1 \mathrm{ng}$ DNA, $120 \mathrm{nM}$ ADP, $80 \mathrm{nM}$ ICP, $100 \mathrm{nM}$ PCR primers, $4 \%$ DMSO (by volume), and $1 \times$ Master Mix (Applied Biosystems). Amplification was carried out by incubation for $2 \mathrm{~min}$ at $50^{\circ} \mathrm{C}, 10 \mathrm{~min}$ at $95^{\circ} \mathrm{C}$, then 40 cycles at $96^{\circ} \mathrm{C}$ for $15 \mathrm{~s}$, and $62.5^{\circ} \mathrm{C}$ for $90 \mathrm{~s}$. Genotypes were generated using ABIPRISM 7700 Sequence Detection system software. On each plate, previously sequenced genomic DNA samples were used as standards for SS-, LS-, and LL-genotypes.

\section{RESULTS}

\section{Biochemical Results}

A 2 (drug group: tryp- vs placebo) by 2 (timepoint: baseline $v s 5 \mathrm{~h}$ postcapsules) ANOVA was conducted on serum-free tryptophan levels (see Table 1). This revealed a main effect of drug group (tryp- or placebo) $(\mathrm{F}(1,33)=8.94, p=0.005)$, as well as a significant drug group by time point interaction $(\mathrm{F}(1,33)=21.41, p<0.001)$. Examination of this interaction with follow-up ANOVAs demonstrated there were no significant differences in the baseline-free tryptophan levels between the drug groups $(\mathrm{F}(1,33)<1, p=0.9)$ but there was a significant reduction in free tryptophan levels in the tryp- group $5 \mathrm{~h}$ following capsule ingestion $(\mathrm{F}(1,33)=40.50, p<0.001)(\mathrm{M}[$ free trypin $\mu \mathrm{g} / \mathrm{ml}]=0.213, \quad \mathrm{SE}=0.05 ; \quad \mathrm{M}[$ free placebo $]=0.658]$, $\mathrm{SE}=0.05)$. This represented a reduction of $80 \%$ in free tryptophan levels in the tryp- group compared to a $36 \%$ reduction in the placebo group. Similarly, there were no significant differences between groups at baseline in the LNAA ratio $(\mathrm{F}(1,33)<1, p=0.8)$, while at $5 \mathrm{~h}$ postcapsule ingestion, there was a significant reduction of the LNAA ratio in the tryp- group compared to the placebo group

Table I Baseline and 5 Hour Free Tryptophan and tryp/LNAA Serum Levels

\begin{tabular}{|c|c|c|c|c|}
\hline Group & Baseline free tryptophan & $5 \mathrm{~h}$ free tryptophan & Baseline tryp/LNAA & $5 \mathrm{hr}$ tryp/LNAA \\
\hline \multicolumn{5}{|c|}{ Tryptophan depleted } \\
\hline Total & $1.04(0.08)$ & $0.21(0.04)$ & $0.17(0.03)$ & $0.02(0.01)$ \\
\hline Male/female & I. $10 / 1.00(0.1)$ & $0.23 / 0.20(0.8)$ & $0.09 / 0.23(0.03)$ & $0.02 / 0.02(0.01)$ \\
\hline LL & $1.12(0.14)$ & $0.18(0.09)$ & $0.18(0.06)$ & $0.02(0.02)$ \\
\hline S-carriers & $1.04(0.10)$ & $0.25(0.07)$ & $0.21(0.04)$ & $0.03(0.01)$ \\
\hline Total & $1.03(0.07)$ & $0.66(0.05)$ & $0.18(0.01)$ & $0.07(0.01)$ \\
\hline Male/female & $1.03 / 1.02(0.10)$ & $0.70 / 0.62(0.07)$ & $0.17 / 0.18(0.03)$ & $0.08 / 0.06(0.10)$ \\
\hline LL & $1.09(0.11)$ & $0.63(0.07)$ & $0.19(0.04)$ & $0.07(0.01)$ \\
\hline S-carriers & $0.90(0.11)$ & $0.76(0.07)$ & $0.14(0.04)$ & $0.07(0.01)$ \\
\hline
\end{tabular}

Serum-free tryptophan levels in $\mu \mathrm{g} / \mathrm{ml}$. SE in parenthesis. 
$(\mathrm{F}(1,33)=17.40, p<0.001)$. Based on prior reports of differential gender effects of TD (Harmer et al, 2003; Nishizawa et al, 1997), 2 (gender) $\times 2$ (drug group) $\times 2$ (baseline $v s 5 \mathrm{~h}$ ) ANOVAs were conducted on the free tryptophan levels and tryp/LNAA ratios. These revealed no significant gender main effect or interaction on free tryptophan levels, but did reveal a significant gender $\times$ drug group interaction on tryp/LNAA ratios $(\mathrm{F}(1,31)=4.33$; $p<0.05$ (see Table 1). Examination of this interaction demonstrated lower baseline levels of the tryp/LNAA ratio in male participants who would undergo TD. Inclusion of gender as an additional between-subjects variable did not demonstrate any significant interactions with drug group in the analysis of passive avoidance or probabilistic response reversal performance. To preserve power, the subsequent analysis reported below did not include gender as a variable.

\section{5-HTTLPR Results}

Genetic samples were available for 26 of the 35 participants due to technical difficulties (14 who received placebo and 12 who received tryp-). As the presence of one or two copies of the S-allele has been associated with decreased 5-HTT mRNA expression compared to the LL-homozygous state (Bradley et al, 2005; Heils et al, 1996; Lesch et al, 1996), to maintain sample size individuals with one $(n=13)$ or two copies $(n=2)$ of the S-allele were grouped together for the analysis and compared to LL-homozygous participants $(n=11)$.

ANOVAs revealed there were no significant differences in age, IQ scores or VAS ratings between the LL- $v s$ S-carrier groups. A 2 (genotype: LL- $v s$ S-carrier) $\times 2$ (drug group: tryp - or placebo) ANOVA conducted on tryptophan levels demonstrated no significant effect of genotype on baseline or $5 \mathrm{~h}$ postcapsule-free tryptophan levels or LNAA ratios and no significant genotype by drug group interactions (see Table 1).

\section{Passive Avoidance Learning}

The passive avoidance error rate was defined as the number of times a participant responded to an $\mathrm{S}-$ (and was thus punished). The omission error rate was equal to the number of times a participant failed to respond to an $\mathrm{S}+$ (and thus failed to obtain a reward). A one-way ANOVA conducted on omission errors in the first block revealed no significant differences between the tryptophan-depleted and placebo groups $(\mathrm{F}(1,33)<1 ; p=0.7)$; mean block 1 omission errors $[$ tryp- $]=2.06$ and $[$ placebo] $=1.89$. A one-way ANOVA conducted on passive avoidance errors revealed no significant differences between the drug groups $(\mathrm{F}(1,33)$ $<1, p=0.9$ ); mean block 1 passive avoidance errors [tryp -$]-=3.87$ and [placebo] $=3.89$. Following Newman and Kosson (1986) initial presentations of stimuli were treated as learning trials, so the first block of responses were excluded from analysis. Main behavioral effects are presented for the entire cohort $(n=35)$ followed by genotype effects and interactions $(n=26)$.

Main behavioral effects. A 2 (drug group: tryp- vs placebo) by 9 (block) ANOVA was conducted on the omission error data. This revealed a main effect for group
$(\mathrm{F}(1,33)=3.023 ; p<0.05$ one-tailed $)$, demonstrating that participants who underwent TD committed significantly more omission errors than those who received placebo: mean omission errors [tryp-] $=2.23, \mathrm{SE}=0.30$; mean omission errors[placebo] $=1.52, \mathrm{SE}=0.28$ (see Figure 1a). A second 2 (drug group: tryp- vs placebo) by 9 (block) ANOVA was conducted on the passive avoidance error data. This revealed no significant main effect for drug group $(\mathrm{F}(1,33)<1 ; p=0.9)$, but did reveal a significant main effect for block $(\mathrm{F}(1,33)=55.93, p<0.001)$; subjects made
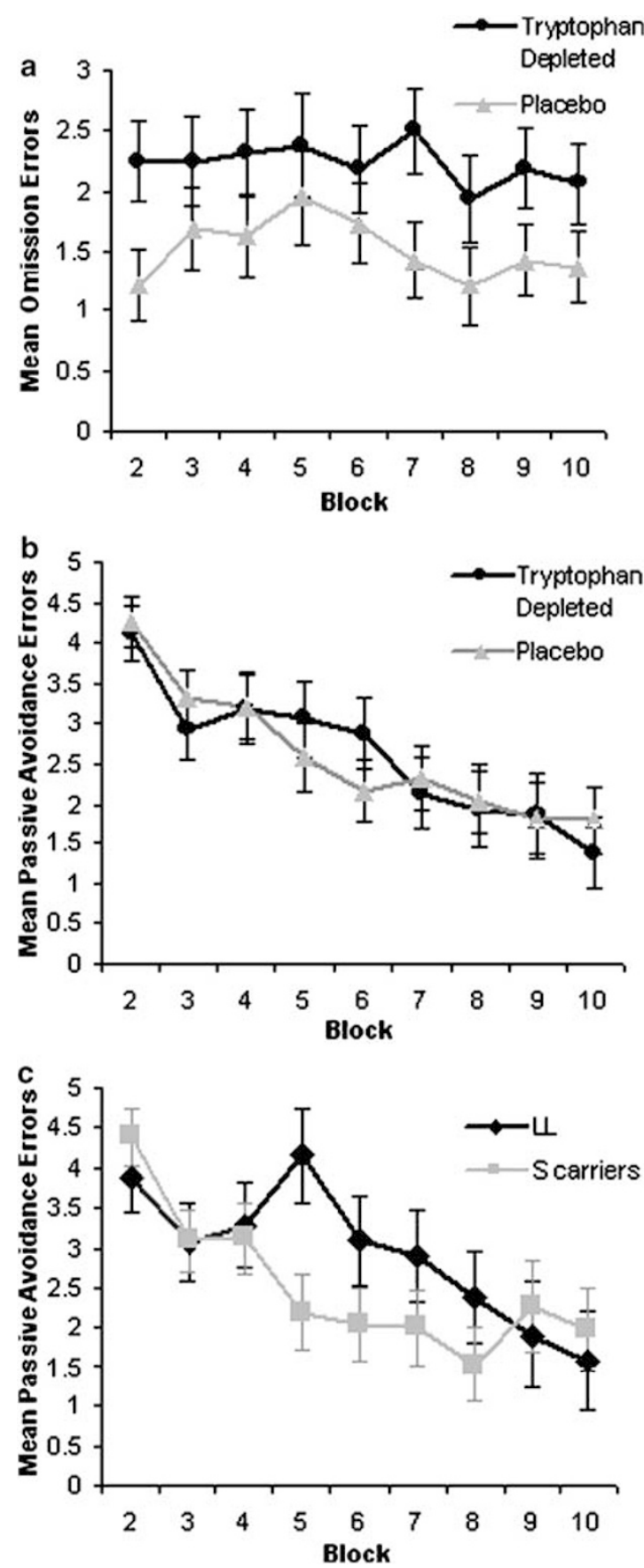

Figure I Passive avoidance learning: (a) mean omission errors and (b) passive avoidance errors per block in tryptophan-depleted and placebo groups; (c) mean passive avoidance errors by block according to 5-HTTLPR genotype. 
fewer passive avoidance errors as the blocks progressed (see Figure $1 b$ ).

Effects of genotype. A 2 (genotype: LL- homozygous vs S-carriers) by 2 (drug group: tryp- vs placebo) by 9 (block) ANOVA on omission errors was conducted on the sample of volunteers with genotype data available $(n=26)$. This revealed no significant main effect of genotype $(F(1,22)$ $<1, p=0.9)$ nor genotype by drug group interactions. A second 2 (genotype: LL-homozygous $v s$ S-carriers) by 2 (drug group: tryp - vs placebo) by 9 (block) ANOVA was conducted on passive avoidance errors. This revealed a significant genotype by block interaction $(\mathrm{F}(8,176)=2.81$, $p<0.05)$. As can be seen in Figure 1c, though the two groups ultimately made similar numbers of errors, the LL-homozygous participants were slower to learn to avoid the 'bad' stimuli than the S-carriers. The S-carriers passive avoidance error rate plateaued by block 5 and beyond, while examination of the passive error rate in the LL-homozygous demonstrates a negative slope indicative of further learning in blocks 5-10. There were no significant drug group by genotype interactions on passive avoidance errors.
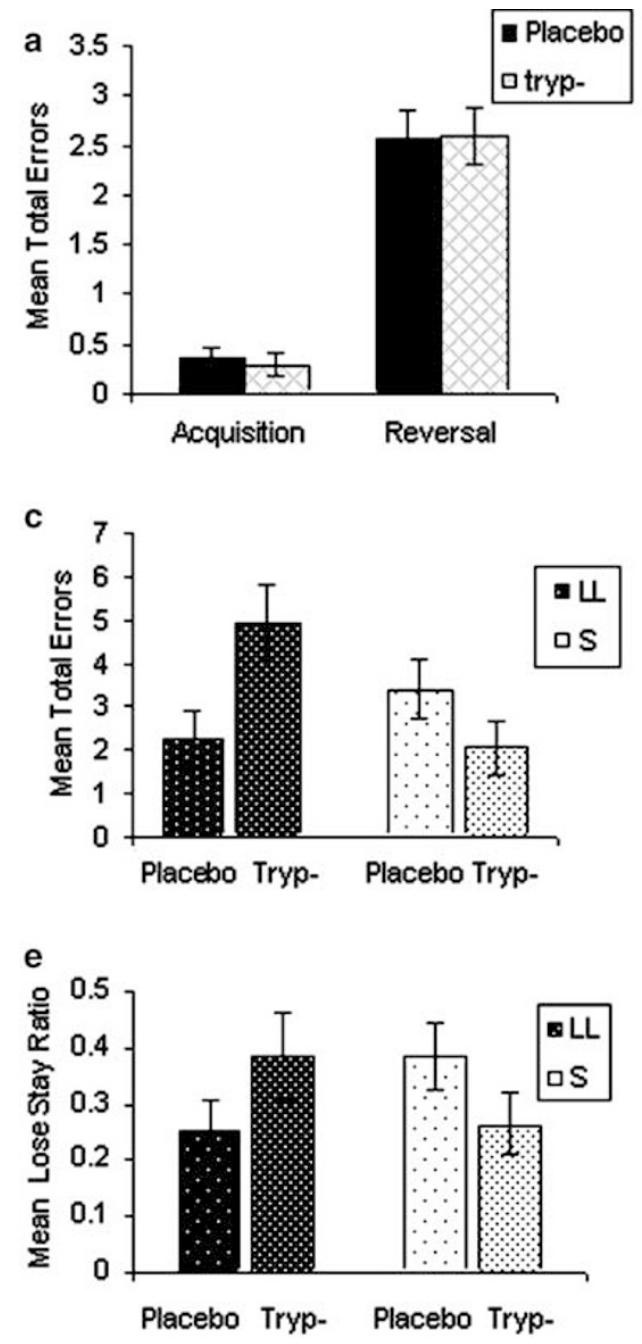

\section{Response Reversal}

Main behavioral effects. All participants successfully reached the learning criteria of 6 consecutive correct responses in the acquisition phase for all pairs and contingencies. A 2 (drug group: tryp $-v s$ placebo) $\times 2$ (contingency: 100:0 vs $80: 20$ ) $\times 2$ (phase: acquisition $v s$ reversal) ANOVA was conducted on total errors. This revealed no main effect of drug group $(\mathrm{F}(1,33)<1, p=0.9)$, $\mathrm{M}$ [tryp - total errors] $=3.53$, SE 0.57 ; $\mathrm{M}$ [placebo total errors] $=3.46$, SE 0.53; (see Figure 2a and b), nor any significant interactions with drug group. There were significant main effects of phase $(\mathrm{F}(1,29)=27.10 ; p<0.01)$ and contingency $(\mathrm{F}(1,29)=35.45 ; p<0.01)$.

Subsequently, three 2 (phase: acquisition $v s$ reversal) by 2 (drug group: tryp $-v s$ placebo) ANOVAs were conducted, each examining specific types of error. The first was on win shift errors (after a correct response that is rewarded, the participant incorrectly shifts to the incorrect response on the subsequent trial). The second was on lose stay responses (after an incorrect response that is punished, the participant still stays with the incorrect response on the subsequent trial). The third was on win maintenance failures (after a
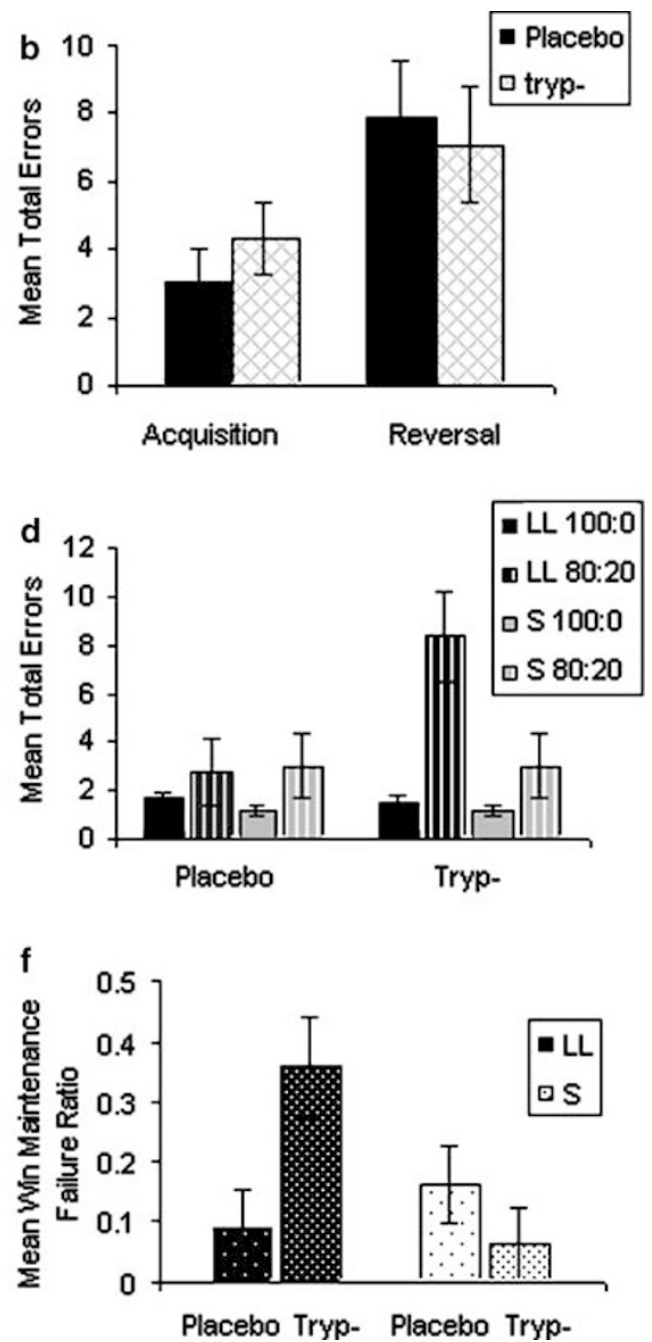

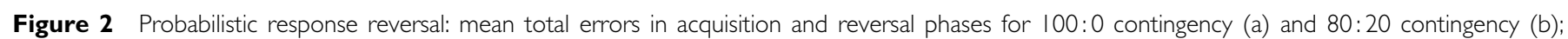

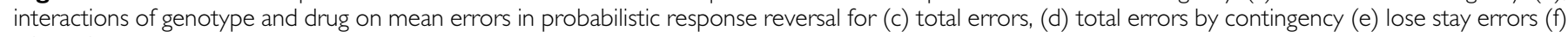
win maintenance errors. 
correct response that was punished, the participant fails to maintain the correct response and incorrectly shifts to the incorrect response) (Murphy et al, 2003). These three ANOVAs failed to reveal any significant group differences or phase by drug group interactions $(\mathrm{F}(1,33)<1, p=0.9$; $p=0.9 ; p=0.8$ respectively).

\section{Effects of Genotype}

To analyze genotype effects on response reversal task performance, a 2 (genotype: LL vs S-carrier) by 2 (drug group: tryp- or placebo) by 2 (phase) by 2 (contingency) repeated measures ANOVA was conducted on mean errors for the participants with available genotype information $(n=26)$. This revealed a significant drug by genotype interaction $(\mathrm{F}(1,22)=7.69, p<0.05)$ well as a contingency by drug by genotype interaction $(\mathrm{F}(1,22)=6.63, p<0.05)$. Follow-up ANOVAs to investigate this interaction revealed that the LL-homozygotes who underwent TD committed significantly more errors than the S-carriers who underwent $\operatorname{TD}(\mathrm{F}(1,10)=9.90, p<0.05)$, and more than the LL-homozygotes who received placebo $(\mathrm{F}(1,10)=9.17$, $p<0.05)$, particularly for stimuli with the probabilistic $(80: 20)$ feedback contingency (see Figure $2 c$ and $d)$.

Three 2 (genotype: LL-homozygous $v s \quad \mathrm{~S}$-carrier) $\times 2$ (drug group: tryp $-v s$ placebo) $\times 2$ (phase: acquisition $v s$ reversal) ANOVAs examined the three response reversal error types. The ANOVA conducted on the win shift errors revealed no significant effects or interactions. The ANOVA conducted on the lose stay errors demonstrated a strong trend for a drug by genotype interaction $(\mathrm{F}(1,22)=4.06$, $p<0.06$ ) (see Figure 2e). Examination of this interaction revealed a trend for LL-homozygotes who were tryptophandepleted to commit more lose stay errors (to follow an incorrect response with negative feedback with another incorrect response to the same stimulus) than LL-homozygous participants who received placebo $(\mathrm{F}(1,10)=4.38$, $p=0.07)$. In contrast, there were no significant differences in lose stay errors between the S-carrier placebo $v s$ trypgroups. The ANOVA conducted on the win maintenance errors revealed a significant drug by genotype interaction $(\mathrm{F}(1,22)=7.11, p=0.01)$. The LL-homozygous tryptophandepleted individuals were significantly less likely to maintain the correct response in the face of probabilistic punishment than both the S-carrier tryptophan-depleted group $(\mathrm{F}(1,10)=9.15, p<0.05)$ or the LL-homozygous placebo groups $(F(1,10)=6.17, p<0.05)$ (see Figure $2 f$ ).

\section{DISCUSSION}

Here, we report the effects of $\mathrm{TD}$ and associations of 5-HTTLPR long and short variants on the passive avoidance and response reversal learning tasks. Passive avoidance learning requires learning to approach the 'good' stimuli that engender reward and avoid the 'bad' stimuli that engender punishment (Baxter and Murray, 2002). When we analyzed the entire cohort together, we found that participants who underwent TD committed more omission errors, or failures to respond to a rewarded stimulus, than the control group. This effect could not be ascribed to a generalized lack of responding, as tryptophan-depleted participants committed an equal number of erroneous responses to punished stimuli as compared to participants who received placebo. In contrast, when looking at the effects of TD on response reversal learning, we found no significant effects. However, when the 5-HTTLPR genotype was included as a factor in the analysis, several genotypespecific effects and genotype interactions with TD were revealed. Our results suggest that TD alters aspects of reward processing independent of 5-HTTLPR genotype, while other components of passive avoidance learning and response reversal performance during TD are influenced by 5-HTTLPR status or interactions with TD. Together these findings extend prior studies examining the affects of TD on various aspects of cognition and affect (Clark et al, 2005; Evers et al, 2005; Murphy et al, 2003; Neumeister, 2003; Park et al, 1994; Rogers et al, 1999, 2003). Additionally, and perhaps most importantly, this study demonstrates the powerful role genotypes may exert on dissociable components of cognitive and emotional tasks and on the response to pharmacologic challenges. In doing so, it suggests one potential cause for conflicting results from prior studies during TD.

The demonstration that tryptophan-depleted participants failed to respond adequately to rewarded stimuli during the passive avoidance task is in line with prior reports that TD has effects on various types of reward related processing (Cools et al, 2005a; Murphy et al, 2003; Rogers et al, 1999). In contrast to our findings of impaired stimulus-reward reinforcement learning, we did not find any significant differences in stimulus-punishment processing between the tryptophan-depleted and control groups. Here, we have demonstrated that TD induces a dissociable effect on stimulus-reward learning $v s$ stimulus-punishment learning. This dissociation held even when 5-HTTLPR genotype was included in the analysis. The preservation of stimuluspunishment responses is in contrast to earlier theories of a role of serotonin in the processing of aversive stimuli (cf. Rogers et al, 1999; Tye et al, 1977; Wilkinson et al, 1995). However, the absence of effects of TD on stimuluspunishment processing is in accord with other more recent reports showing no differential effects of punishment information on decision making tasks (Anderson et al, 2003; Rogers et al, 2003).

Given findings of slower response latencies in prior tryptophan-depletion studies, the possibility of slower response latencies contributing to increased omission errors in the passive avoidance task is an important consideration. Reaction time (RT) data were not available from the passive avoidance task used in this study. Examination of RT latencies in other tryptophan-depletion studies demonstrates between group average RT differences from 10 to $330 \mathrm{~ms}$ with stimulus/response windows from 1200 to $2000 \mathrm{~ms}$ (Cools et al, 2005a; Evers et al, 2005; Murphy et al, 2003). As these differences did not correspond to increases in omissions, coupled with the longer stimulus/response interval in the present study $(3000 \mathrm{~ms})$ we suggest it is unlikely differences in reaction times led to the increased omission errors in the passive avoidance task.

As serotonergic innervation is widespread in the rat (Steinbusch, 1981), non-human primate (Wilson and Molliver, 1991), and human brains (Pazos et al, 1987a, b), 
there are several candidate brain regions which may be responsible for the impaired passive avoidance learning described here. Based on animal studies, both stimulusreward and stimulus-punishment learning recruit the amygdala, striatum and middle frontal cortex (Baxter and Murray, 2002; Everitt et al, 1987; Seymour et al, 2004). Cell recordings in rats demonstrate the presence of neurons with selective responding to cues predictive of punishment and others to reward in both the amygdala and orbitofrontal cortex (Schoenbaum et al, 1998, 2002; Setlow et al, 2002). Functional MRI of passive avoidance learning demonstrated recruitment of the anterior cingulate, middle frontal gyrus, right posterior cingulate, left parietal cortex, hippocampus, caudate, and amygdala during both reward and punishment learning (Newman and Kosson, 1986). All of these regions are innervated by serotonergic axons, therefore combining neurochemical manipulations such as TD with fMRI imaging during the passive avoidance and other instrumental learning tasks will be necessary to further characterize the dissociable stimulus-reward and stimuluspunishment reinforcement learning systems in humans.

Our initial analysis which suggested a lack of effect of TD on probabilistic response reversal performance appeared consistent with several others studies showing no significant behavioral effect on response reversal errors during TD (Evers et al, 2005; Talbot et al, 2005). However, the subsequent integration of the 5-HTTLPR genotype in the analysis revealed several effects of TD on probabilistic response reversal learning performance. These effects were seen in the tryptophan-depleted LL-homozygous group who committed more total errors, increased lose-stay errors, and increased win maintenance failures across the acquisition and reversal phases. It should be noted, however, that the total number of errors committed by both groups in the probabilistic response reversal task was relatively low, thus limiting interpretation of the component error types. In concert with the demonstration that the LLhomozygous group was slower to learn to avoid stimuli associated with punishment in the passive avoidance task, these findings suggest an impairment in tryptophandepleted LL-homozygotes in the utilization of negative feedback, whether true or probabilistic, to guide appropriate responding. In vitro studies of 5-HTTLPR function have demonstrated increased reuptake of extracellular serotonin in platelets by the LL-homozygous status compared to the SS- or SL-variants (Greenberg et al, 1999; Lesch et al, 1996). The acute interactions found here suggests that with respect to the utilization of punishment information, long allele homozygotes are more sensitive to diminutions in serotonin during TD, possibly due in part to their accelerated serotonin reuptake compared to short allele carriers. Interpretation of the genetic results may be limited by recent identification of another locus of allelic variation in the 5-HTTLPR with functional ramifications (Hu et al, 2006). As the current study was not powered to account for the triallelic model, future investigations of 5-HTTLPR genotype and serotonin interactions will be necessary to further explore these findings.

Of note, interpretation of the negative results in this study may be limited by the amino-acid mixture and control preparation of lactose used. Although the dosage of amino acids was lower than that used in many other studies, it did produce relative reductions in free tryptophan and the tryp/ LNAA ratio comparable to that of other studies of response reversal (Evers et al, 2005; Rogers et al, 1999; Talbot et al, 2005). However, we also observed relatively greater decreases in these parameters in our control group than observed in these studies (Evers et al, 2005; Rogers et al, 1999; Talbot et al, 2005). This may have resulted from the use of lactose capsules as the placebo, coupled with the ingestion of low-tryptophan meals, producing a decrease in the tryp/LNAA ratio in the control group. The relative reduction in group differences at the biochemical level is an important consideration in the interpretation of our negative results. Further studies would be strengthened by use of a control mixture containing tryptophan to avoid generation of a non-neutral control condition.

The finding that some individuals are resilient while others are susceptible to TD-induced deficits during probabilistic response reversal offers one possible explanation for the variability in results of earlier studies. Future studies employing pharmacologic manipulations in healthy volunteers designed to assess the interactions with relevant genotypes will likely unmask further important effects and interactions.

\section{ACKNOWLEDGEMENTS}

This research was supported by the Intramural Research Program of the NIH: NIMH.

\section{REFERENCES}

Ambrogi Lorenzini CG, Baldi E, Bucherelli C, Sacchetti B, Tassoni G (1997). Role of ventral hippocampus in acquisition, consolidation and retrieval of rat's passive avoidance response memory trace. Brain Res 768: 242-248.

Ambrogi Lorenzini CG, Baldi E, Bucherelli C, Sacchetti B, Tassoni G (1999). Neural topography and chronology of memory consolidation: a review of functional inactivation findings. Neurobiol Learn Mem 71: 1-18.

Anderson IM, Richell RA, Bradshaw CM (2003). The effect of acute tryptophan depletion on probabilistic choice. J Psychopharmacol 17: 3-7.

Baxter MG, Murray EA (2002). The amygdala and reward. Nat Rev Neurosci 3: 563-573.

Bermudez-Rattoni F, McGaugh JL (1991). Insular cortex and amygdala lesions differentially affect acquisition on inhibitory avoidance and conditioned taste aversion. Brain Res 549: 165-170.

Blair RJR, Mitchell DGV, Leonard A, Budhani S, Peschardt KS, Newman C (2004). Passive avoidance learning in psychopathic individuals: modulation by reward but not by punishment. Pers Individual Differ 37: 1179-1192.

Bradley SL, Dodelzon K, Sandhu HK, Philibert RA (2005). Relationship of serotonin transporter gene polymorphisms and haplotypes to mRNA transcription. Am J Med Genet B Neuropsychiatr Genet 136: 58-61.

Budhani S, Blair RJ (2005). Response reversal and children with psychopathic tendencies: success is a function of salience of contingency change. J Child Psychol Psychiatry 46: 972-981.

Canli T, Omura K, Haas BW, Fallgatter A, Constable RT, Lesch KP (2005). Beyond affect: a role for genetic variation of the serotonin transporter in neural activation during a cognitive attention task. Proc Natl Acad Sci USA 102: 12224-12229. 
Carpenter LL, Anderson GM, Pelton GH, Gudin JA, Kirwin PD, Price LH et al (1998). Tryptophan depletion during continuous CSF sampling in healthy human subjects. Neuropsychopharmacology 19: 26-35.

Chamberlain SR, Muller U, Blackwell AD, Clark L, Robbins TW, Sahakian BJ (2006). Neurochemical modulation of response inhibition and probabilistic learning in humans. Science 311: 861-863.

Clark L, Roiser JP, Cools R, Rubinsztein DC, Sahakian BJ, Robbins TW (2005). Stop signal response inhibition is not modulated by tryptophan depletion or the serotonin transporter polymorphism in healthy volunteers: implications for the 5-HT theory of impulsivity. Psychopharmacology (Berlin) 182: 570-578.

Clarke HF, Dalley JW, Crofts HS, Robbins TW, Roberts AC (2004). Cognitive inflexibility after prefrontal serotonin depletion. Science 304: 878-880.

Clarke HF, Walker SC, Crofts HS, Dalley JW, Robbins TW, Roberts AC (2005). Prefrontal serotonin depletion affects reversal learning but not attentional set shifting. J Neurosci 25: 532-538.

Cools R, Blackwell A, Clark L, Menzies L, Cox S, Robbins TW (2005a). Tryptophan depletion disrupts the motivational guidance of goal-directed behavior as a function of trait impulsivity. Neuropsychopharmacology 30: 1362-1373.

Cools R, Calder AJ, Lawrence AD, Clark L, Bullmore E, Robbins TW (2005b). Individual differences in threat sensitivity predict serotonergic modulation of amygdala response to fearful faces. Psychopharmacology (Berlin) 180: 670-679.

Cools R, Clark L, Owen AM, Robbins TW (2002). Defining the neural mechanisms of probabilistic reversal learning using event-related functional magnetic resonance imaging. J Neurosci 22: 4563-4567.

Everitt BJ, Robbins TW, Evenden JL, Marston HM, Jones GH, Sirkia TE (1987). The effects of excitotoxic lesions of the substantia innominata, ventral and dorsal globus pallidus on the acquisition and retention of a conditional visual discrimination: implications for cholinergic hypotheses of learning and memory. Neuroscience 22: 441-469.

Evers EA, Cools R, Clark L, van der Veen FM, Jolles J, Sahakian BJ et al (2005). Serotonergic modulation of prefrontal cortex during negative feedback in probabilistic reversal learning. Neuropsychopharmacology 30: 1138-1147.

Gallagher M, McMahan RW, Schoenbaum G (1999). Orbitofrontal cortex and representation of incentive value in associative learning. J Neurosci 19: 6610-6614.

Garpenstrand H, Annas P, Ekblom J, Oreland L, Fredrikson M (2001). Human fear conditioning is related to dopaminergic and serotonergic biological markers. Behav Neurosci 115: 358-364.

Gessa GL, Biggio G, Fadda F, Corsini GU, Tagliamonte A (1975). Tryptophan-free diet: a new means for rapidly decreasing brain tryptophan content and serotonin synthesis. Acta Vitaminol Enzymol 29: 72-78.

Greenberg BD, Tolliver TJ, Huang SJ, Li Q, Bengel D, Murphy DL (1999). Genetic variation in the serotonin transporter promoter region affects serotonin uptake in human blood platelets. $\mathrm{Am} \mathrm{J}$ Med Genet 88: 83-87.

Hariri AR, Drabant EM, Munoz KE, Kolachana BS, Mattay VS, Egan MF et al (2005). A susceptibility gene for affective disorders and the response of the human amygdala. Arch Gen Psychiatry 62: 146-152.

Hariri AR, Holmes A (2006). Genetics of emotional regulation: the role of the serotonin transporter in neural function. Trends Cogn Sci 10: 182-191.

Hariri AR, Mattay VS, Tessitore A, Kolachana B, Fera F, Goldman $\mathrm{D}$ et al (2002). Serotonin transporter genetic variation and the response of the human amygdala. Science 297: 400-403.

Harmer CJ, Mackay CE, Reid CB, Cowen PJ, Goodwin GM (2006). Antidepressant drug treatment modifies the neural processing of nonconscious threat cues. Biol Psychiatry 59: 816-820.
Harmer CJ, Rogers RD, Tunbridge E, Cowen PJ, Goodwin GM (2003). Tryptophan depletion decreases the recognition of fear in female volunteers. Psychopharmacology (Berlin) 167: 411-417.

Harmer CJ, Shelley NC, Cowen PJ, Goodwin GM (2004). Increased positive versus negative affective perception and memory in healthy volunteers following selective serotonin and norepinephrine reuptake inhibition. Am J Psychiatry 161: 1256-1263.

Heils A, Teufel A, Petri S, Stober G, Riederer P, Bengel D et al (1996). Allelic variation of human serotonin transporter gene expression. J Neurochem 66: 2621-2624.

Heinz A, Braus DF, Smolka MN, Wrase J, Puls I, Hermann D et al (2005). Amygdala-prefrontal coupling depends on a genetic variation of the serotonin transporter. Nat Neurosci 8: 20-21.

Hu X-Z, Lipsky RH, Zhu G, Akhtar LA, Taubman J, Greenberg BD et al (2006). Serotonin transporter promoter gain-of-function genotypes are linked to obsessive-compulsive disorder. $\mathrm{Am} \mathrm{J}$ Hum Genet 78: 815-826.

Kosson DS, Budhani S, Nakic M, Chen G, Saad ZS, Vythilingam M et al (2006). The role of the amygdala and rostral anterior cingulate in encoding expected outcomes during learning. Neuroimage 29: 1161-1172.

Kringelbach ML, Rolls ET (2003). Neural correlates of rapid reversal learning in a simple model of human social interaction. Neuroimage 20: 1371-1383.

Lesch KP, Bengel D, Heils A, Sabol SZ, Greenberg BD, Petri S et al (1996). Association of anxiety-related traits with a polymorphism in the serotonin transporter gene regulatory region. Science 274: 1527-1531.

McGaugh JL (2002). Memory consolidation and the amygdala: a systems perspective. Trends Neurosci 25: 456.

Murphy FC, Michael A, Robbins TW, Sahakian BJ (2003). Neuropsychological impairment in patients with major depressive disorder: the effects of feedback on task performance. Psychol Med 33: 455-467.

Neumeister A (2003). Tryptophan depletion, serotonin, and depression: where do we stand? Psychopharmacol Bull 37: 99-115.

Neumeister A, Nugent AC, Waldeck T, Geraci M, Schwarz M, Bonne $\mathrm{O}$ et al (2004). Neural and behavioral responses to tryptophan depletion in unmedicated patients with remitted major depressive disorder and controls. Arch Gen Psychiatry 61: 765-773.

Newman JP, Kosson DS (1986). Passive avoidance learning in psychopathic and nonpsychopathic offenders. J Abnorm Psychol 95: $252-256$.

Nishizawa S, Benkelfat C, Young SN, Leyton M, Mzengeza S, de Montigny C et al (1997). Differences between males and females in rates of serotonin synthesis in human brain. PNAS 94: $5308-5313$.

O'Doherty J, Critchley H, Deichmann R, Dolan RJ (2003). Dissociating valence of outcome from behavioral control in human orbital and ventral prefrontal cortices. J Neurosci 23: 7931-7939.

Park SB, Coull JT, McShane RH, Young AH, Sahakian BJ, Robbins TW et al (1994). Tryptophan depletion in normal volunteers produces selective impairments in learning and memory. Neuropharmacology 33: 575-588.

Pazos A, Probst A, Palacios JM (1987a). Serotonin receptors in the human brain - III. Autoradiographic mapping of serotonin-1 receptors. Neuroscience 21: 97-122.

Pazos A, Probst A, Palacios JM (1987b). Serotonin receptors in the human brain-IV. Autoradiographic mapping of serotonin-2 receptors. Neuroscience 21: 123-139.

Perez-Cruet J, Chase TN, Murphy DL (1974). Dietary regulation of brain tryptophan metabolism by plasma ratio of free tryptophan and neutral amino acids in humans. Nature 248: 693-695.

Pezawas L, Meyer-Lindenberg A, Drabant EM, Verchinski BA, Munoz KE, Kolachana BS et al (2005). 5-HTTLPR polymorphism 
impacts human cingulate-amygdala interactions: a genetic susceptibility mechanism for depression. Nat Neurosci 8: 828-834.

Remijnse PL, Nielen MM, Uylings HB, Veltman DJ (2005). Neural correlates of a reversal learning task with an affectively neutral baseline: an event-related fMRI study. Neuroimage 26: 609-618.

Rogers RD, Blackshaw AJ, Middleton HC, Matthews K, Hawtin K, Crowley C et al (1999). Tryptophan depletion impairs stimulusreward learning while methylphenidate disrupts attentional control in healthy young adults: implications for the monoaminergic basis of impulsive behaviour. Psychopharmacology (Berlin) 146: 482-491.

Rogers RD, Tunbridge EM, Bhagwagar Z, Drevets WC, Sahakian BJ, Carter CS (2003). Tryptophan depletion alters the decisionmaking of healthy volunteers through altered processing of reward cues. Neuropsychopharmacology 28: 153-162.

Sandberg K, Sanberg PR, Hanin I, Fisher A, Coyle JT (1984). Cholinergic lesion of the striatum impairs acquisition and retention of a passive avoidance response. Behav Neurosci 98: 162-165.

Schoenbaum G, Chiba AA, Gallagher M (1998). Orbitofrontal cortex and basolateral amygdala encode expected outcomes during learning. Nat Neurosci 1: 155-159.

Schoenbaum G, Nugent SL, Saddoris MP, Setlow B (2002). Orbitofrontal lesions in rats impair reversal but not acquisition of go, no-go odor discriminations. Neuroreport 13: 885-890.

Setlow B, Gallagher M, Holland PC (2002). The basolateral complex of the amygdala is necessary for acquisition but not expression of CS motivational value in appetitive Pavlovian second-order conditioning. Eur J Neurosci 15: 1841-1853.

Seymour B, O’Doherty JP, Dayan P, Koltzenburg M, Jones AK, Dolan RJ et al (2004). Temporal difference models describe higher-order learning in humans. Nature 429: 664-667.

Snodgrass JG, Vanderwart M (1980). A standardized set of 260 pictures: norms for name agreement, image agreement, familiarity, and visual complexity. J Exp Psychol (Hum Learn) 6: 174-215.

Steinbusch HW (1981). Distribution of serotonin-immunoreactivity in the central nervous system of the rat-cell bodies and terminals. Neuroscience 6: 557-618.

Stockmeier CA (1997). Neurobiology of serotonin in depression and suicide. Ann NY Acad Sci 836: 220-232.

Talbot PS, Watson DR, Barrett SL, Cooper SJ (2005). Rapid tryptophan depletion improves decision-making cognition in healthy humans without affecting reversal learning or set shifting. Neuropsychopharmacology 31: 1519-1525.

Treit D, Menard J (1997). Dissociations among the anxiolytic effects of septal, hippocampal, and amygdaloid lesions. Behav Neurosci 111: 653-658.

Tremblay L, Schultz W (2000). Reward-related neuronal activity during go-nogo task performance in primate orbitofrontal cortex. J Neurophysiol 83: 1864-1876.

Tye NC, Everitt BJ, Iversen SD (1977). 5-Hydroxytryptamine and punishment. Nature 268: 741-743.

Wilkinson LS, Humby T, Robbins TW, Everitt BJ (1995). Differential effects of forebrain 5-hydroxytryptamine depletions on Pavlovian aversive conditioning to discrete and contextual stimuli in the rat. Eur J Neurosci 7: 2042-2052.

Williams WA, Shoaf SE, Hommer D, Rawlings R, Linnoila M (1999). Effects of acute tryptophan depletion on plasma and cerebrospinal fluid tryptophan and 5-hydroxyindoleacetic acid in normal volunteers. J Neurochem 72: 1641-1647.

Wilson MA, Molliver ME (1991). The organization of serotonergic projections to cerebral cortex in primates: regional distribution of axon terminals. Neuroscience 44: 537-553.

Wolfe BE, Metzger ED, Jimerson DC (1995). Comparison of the effects of amino acid mixture and placebo on plasma tryptophan to large neutral amino acid ratio. Life Sci 56: 1395-1400.

Zohar J, Kennedy JL, Hollander E, Koran LM (2004). Serotonin-1D hypothesis of obsessive-compulsive disorder: an update. J Clin Psychiatry 65(Suppl 14): 18-21. 